cases. 11 patients had documented anxiety with $72.7 \%$ being treated effectively with atomoxetine. $31 \%$ of patients' had documented side-effects with $16 \%$ of these being tics. $20 \%$ of patient's required augmentation.

Conclusion. The results indicate that the majority of doctors at CYPS in Malta adhered to the NICE guidelines 2018 and atomoxetine was proven to be efficacious as a second line drug in the treatment of ADHD. However, better adherence to NICE guidelines is required when it comes to the calculation of appropriate dosage. Our prediction is had dose recommendations according to weight been adhered to there may have been less side-effects documented.

\section{Audit: lithium monitoring for psychiatric inpatients and community patients during the initiation phase}

Damodar Chari, Mohammed Abbas and Kajal Dhesi

Leicestershire Partnership Trust

${ }^{\star}$ Corresponding author.

doi: 10.1192/bjo.2021.227

Aims. Measure compliance with standards requiring baseline work up before Lithium therapy is commenced and subsequent Lithium level monitoring during the initiation phase

Method. All inpatients and outpatients who were started on Lithium between 2018 and 2019 within the Leicestershire Partnership NHS trust. Case notes were of patients 128 were retrieved from the electronic system and an audit proforma was completed to ascertain adherence to auditing standards as per $\mathrm{BNF}$ and trust guidelines. Parameters monitored were full blood count $(\mathrm{FBC})$, renal functions test including serum electrolytes, thyroid function test, and BMI before commencing Lithium, and serum Lithium periodically after. ECG was needed for those patients with cardiovascular illness. Data were systematically compiled and analyzed descriptively using Microsoft Excel

Result. A total of 128 patients were included in the study. 111 (86.71\%) had FBC, 118 (92.19\%) had renal function test and electrolytes, 114 (89.06\%) had thyroid function test while 99 (77.34\%) had their BMI/weight recorded before initiating Lithium. 26 out of 36 patients with cardiovascular disorder had their ECG recorded. After Lithium was commenced, 108 (84.37\%) had serum Lithium tested a week later, while only 89 (69.53\%) had lithium monitored weekly. Trust guidelines recommend weekly monitoring for up to 4 weeks after a stable dose was reached. This was monitored in only 16 out of 128 patients.

Conclusion. Most of the patients had blood test done before being commenced on Lithium. However it was observed that serum Lithium was not adequately monitored at regular intervals after dose escalations. These finding indicate that there has to be greater awareness of the trust and BNF guidelines with regards to Lithium monitoring.

\section{Hyponatraemia monitoring in those prescribed} antidepressants - an audit from an inpatient older adult ward

Lorena Chavez*, Jake Scott, Richard Hoile, Jane McNulty and Louisa Marchant-Rutherford

Sussex Partnership NHS Foundation Trust

${ }^{\star}$ Corresponding author.

doi: 10.1192/bjo.2021.228

Aims. To assess follow-up of sodium levels for in-patients prescribed antidepressants in practice compare to the standard of 3 monthly sodium levels for all patients who are prescribed antidepressants and at risk of hyponatraemia

Method. A list of the 20 most recently discharged patients from Meridian Ward, an older-adult functional inpatient ward, was prepared by the team administrator on 6th May 2020.

We audited the entire duration of our patient's stay on Meridian Ward (we did not include periods of their admission when they were on other wards) using the electronic notes system, Carenotes.

We also checked the electronic biochemistry results system, ICE, for sodium results, and the discharge summary for mentions of fluid restriction, medications and handover to GP of sodiumchecking. We also checked scanned drug charts to see if they were on antidepressants and other implicated drugs.

For people with episodes of hyponatraemia, in order to retrieve further info we looked at discharge summary and searched the activity notes for the following terms

"Hyponat"

"sodium"

"fluid restrict"

"Low na"

We regarded the following conditions as risk factors for hyponatraemia:

cardiac

malignancy

respiratory

hypothyroid

renal

hepatic

stroke

We regarded following medications as risk factors:

opioids

diuretics

carbamazepine

theophylline

antipsychotics

NSAIDs

PPIs

ACE-I

ARBs

amiodarone

domperidone

sulphonylureas

Result. 14 of the 20 patients were taking antidepressants. Of those: 13 were eligible for regular sodium monitoring due to risk factors 11 of these had 3-monthly sodium levels during admission For only 2 of these did we make a plan for the GP to continue to monitor the sodium level in community 3 had an episode of hyponatraemia implicated antidepressants: sertraline plus mirtazapine mirtazapine (very serious episode which caused seizure) sertraline for 2 of them an appropriate plan was made 1 without a plan - a mild hyponatraemia with nothing documented in the notes

Conclusion. During their admission to Meridian Ward, 85\% of patients taking antidepressants who had risk factors for hyponatraemia had three-monthly sodium levels in line with the trust guidance. However, only two patients (15\%) had a plan for further sodium levels in the discharge summary sent to the GP. This highlights a need for improved awareness of risk factors for hyponatraemia and, in particular, improved communication with general practitioners who are going to take over prescribing of antidepressant medications.

Recommendations

3 monthly $\mathrm{Na}$ levels for all patients with risk factors

i.e. on any antidepressant prescribed PLUS any one of: 